Supplement of Hydrol. Earth Syst. Sci., 22, 5387-5401, 2018

https://doi.org/10.5194/hess-22-5387-2018-supplement

(C) Author(s) 2018. This work is distributed under

the Creative Commons Attribution 4.0 License.

(c) (1)

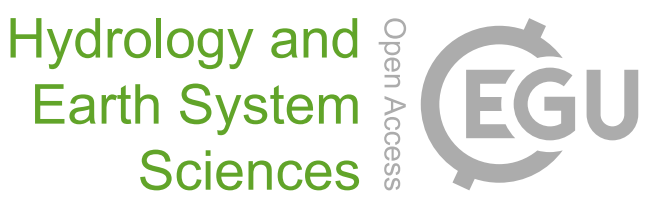

Supplement of

\title{
Future hot-spots for hydro-hazards in Great Britain: a probabilistic assessment
}

\section{Lila Collet et al.}

Correspondence to: Lila Collet (lila.collet@irstea.fr)

The copyright of individual parts of the supplement might differ from the CC BY 4.0 License. 


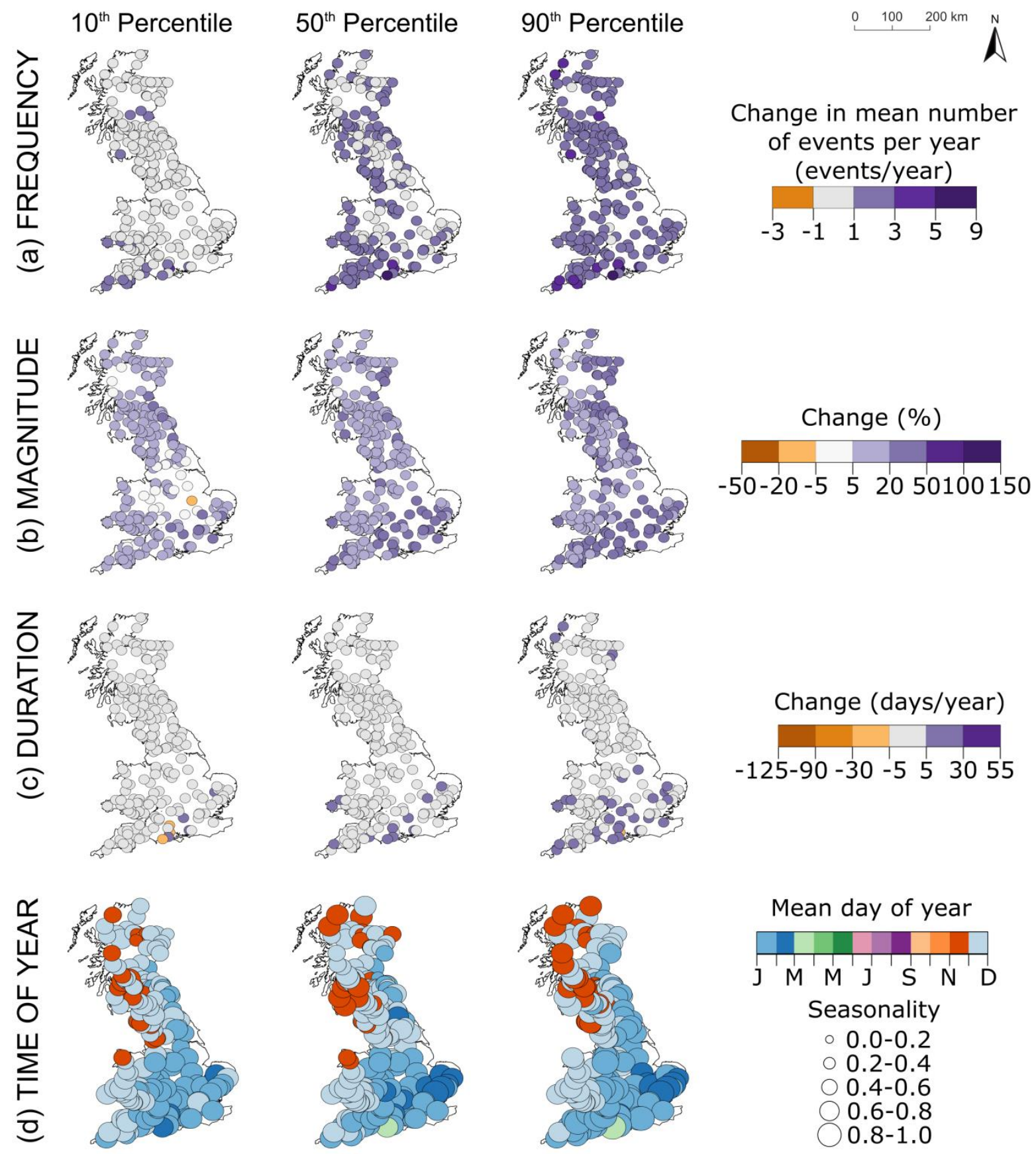

Figure S1: Changes in: (a) frequency, (b) magnitude, (c) duration, (d) and time of year of floods across Great Britain from the baseline to the $2080 \mathrm{~s}$ for the $10^{\text {th }}, 50^{\text {th }}$, and $90^{\text {th }}$ percentiles across the 11 ensemble-members. 

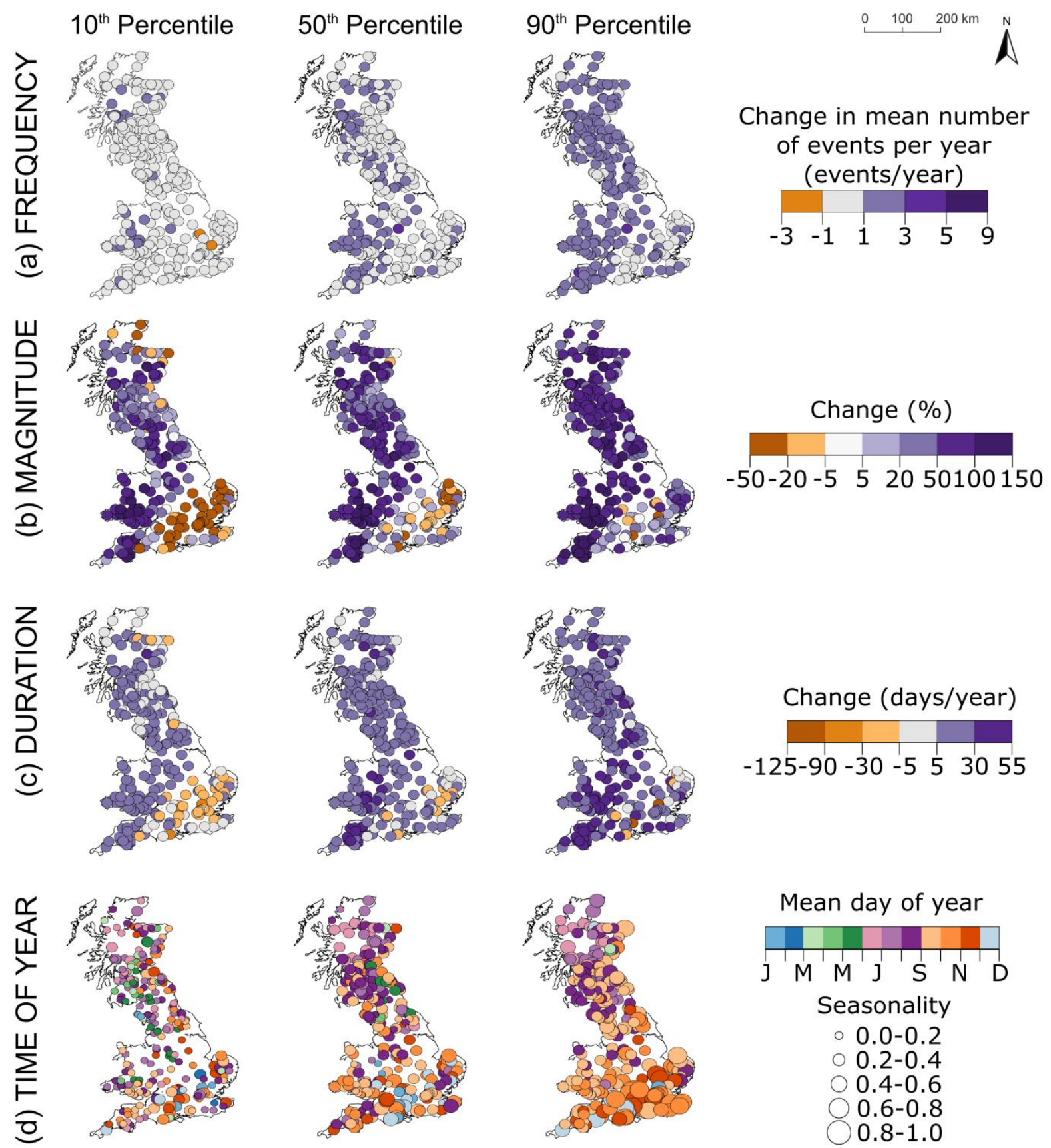
$0.0-0.2$
$00.2-0.4$
0.4-0.6
$0.6-0.8$
$0.8-1.0$

Figure S2: Changes in: (a) frequency, (b) magnitude, (c) duration, and (d) time of year of droughts across Great Britain from the baseline to the $2080 \mathrm{~s}$ for the $10^{\text {th }}, 50^{\text {th }}$, and $90^{\text {th }}$ percentiles across the 11 ensemble-members. 


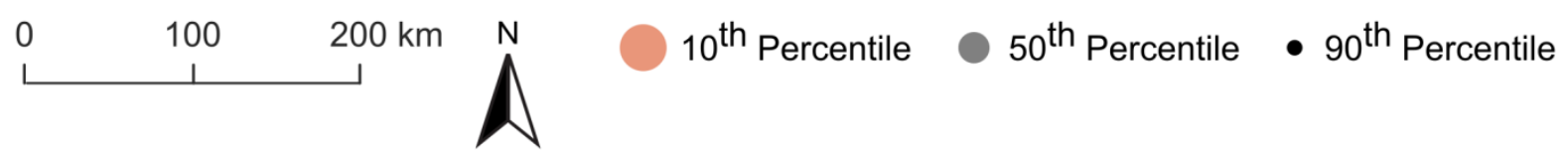
(a) Flood Hot-Spots
(b) Drought Hot-Spots
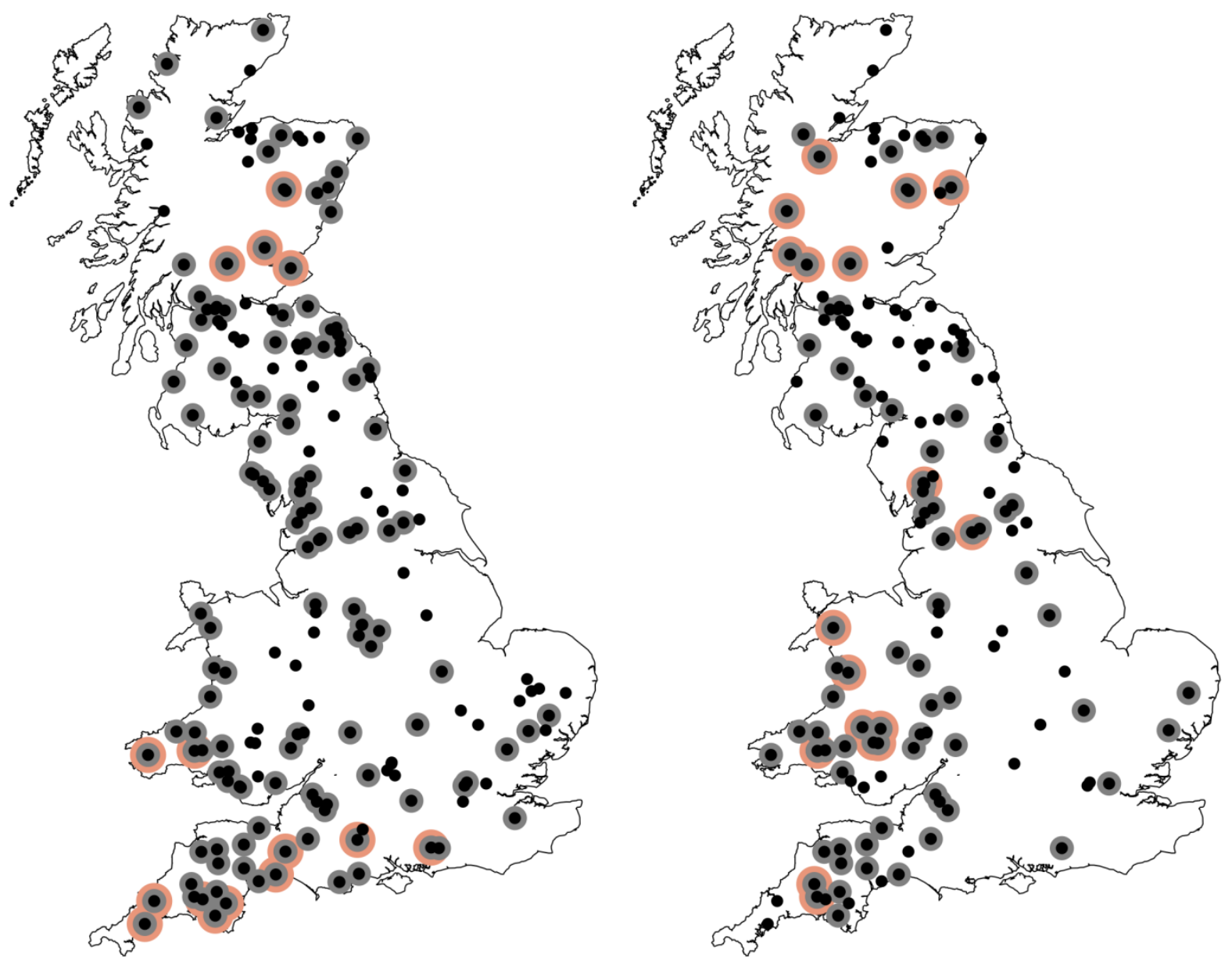

Figure S3: Hot-spots in Great Britain for the $10^{\text {th }}$ (large light red dots), $50^{\text {th }}$ (medium grey dots), and $90^{\text {th }}$ (small black dots) percentiles for (a) flood hazard and (b) drought hazard. 
Time of year and seasonality were defined following the Flood Estimation Handbook (Institute of Hydrology, 1999):

- The day of year $D_{i}$ is the date of the event in the Julian calendar (day of the peak flow for high-flows and day of the minimum flow for low-flows). It can be transformed in an angular value $\theta_{\mathrm{i}}$ on the unit circle $\left(0^{\circ}\right.$ corresponds to the first day of the hydrological year, here starting on the first day of October of each year, $359^{\circ}$ to the last day) (Eq. 1):

$\theta_{i}=\left(D_{i}-0.5\right) \frac{2 \pi}{N D}$

With ND the number of days in the year (ND=365 or 366 for leap years).

The polar coordinate $\bar{\theta}$ of the mean day of year across all the events $\theta_{\mathrm{i}}$ is (Eq. 2):

$\bar{\theta}=\tan ^{-1}\left(\frac{\bar{y}}{\bar{x}}\right)$

where $\quad \bar{x}=\frac{1}{n} \sum_{i=1}^{n} \cos \theta_{i}$

and $\quad \bar{y}=\frac{1}{n} \sum_{i=1}^{n} \sin \theta_{i}$

With $\mathrm{n}$ the number of events in the time period.

The mean day of year MD in Julian days is then (Eq. 3):

$M D=\bar{\theta} \frac{N D}{2 \pi}+0.5$

- The seasonality expresses how concentrated the events are around one period of the year. The higher the number of events during one moment of the year, the higher the seasonality value ( $\bar{r}$ close to 1 ). If the events are spread across the year, the seasonality value $\bar{r}$ is close to 0 . It was computed based on the Cartesian coordinates $\bar{x}$ and $\bar{y}$ of the mean day of year as follow (Eq. 4):

$$
\bar{r}=\sqrt{\bar{x}^{2}+\bar{y}^{2}}
$$

